\title{
Future directions for the European influenza reference laboratory network in influenza surveillance
}

N Goddard (nicholagoddard@hotmail.com) ${ }^{1}$, H Rebelo-de-Andrade ${ }^{2,3}$, A Meijer ${ }^{4}$, J McCauley5 ${ }^{5}$ R Daniels ${ }^{5}$, M Zambon ${ }^{1}$

1. Public Health England (PHE), London, United Kingdom

2. National Health Institute Doctor Ricardo Jorge (INSA) Lisbon, Portugal

3. Faculty of Pharmacy, University of Lisbon, Lisbon, Portugal

4. National Institute for Public Health and the Environment (RIVM), Bilthoven, the Netherlands

5. National Institute of Medical Research (NIMR), London, United Kingdom

Citation style for this article:

Goddard N, Rebelo-de-Andrade H, Meijer A, McCauley J, Daniels R, Zambon M. Future directions for the European influenza reference laboratory network in influenza surveillance. Euro Surveill. 2015;20(30):pii=21195. Available online: http://www.eurosurveillance.org/ViewArticle.aspx?Articleld=21195

By defining strategic objectives for the network of influenza laboratories that have national influenza centre status or national function within European Union Member States, Iceland and Norway, it is possible to align their priorities in undertaking virological surveillance of influenza. This will help maintain and develop the network to meet and adapt to new challenges over the next 3-5 years and underpin a longerterm strategy over 5-10 years. We analysed the key activities undertaken by influenza reference laboratories in Europe and categorised them into a framework of four key strategic objectives areas: enhancing laboratory capability, ensuring laboratory capacity, providing emergency response and translating laboratory data into information for public health action. We make recommendations on the priority areas for future development.

\section{Introduction}

Functional reference laboratory networks capable of undertaking detailed strain characterisation are an important element of communicable disease control. Such networks are major contributors to public health intervention policies through provision of timely and detailed scientific data.

Continuing emergence of influenza viruses emphasises the need for accurate and rapid detection capability and detailed strain characterisation. The influenza $A\left(\mathrm{H}_{1} \mathrm{~N}_{1}\right)$ pdmog pandemic [1], the emergence of influenza $A\left(\mathrm{H}_{3} \mathrm{~N}_{2}\right) \mathrm{V}$ in the United States (US) in 2012 [2] and the more recent emergence of influenza $A\left(\mathrm{H}_{7} \mathrm{Ng}\right)$ [3], influenza $A\left(\mathrm{HgN}_{2}\right)$ [4], influenza $A\left(\mathrm{H}_{1} \mathrm{ON} 8\right)$ [5] and the novel Middle East respiratory syndrome coronavirus (MERS-CoV) infections in humans in 2013 [6] have highlighted the continual threat to human health from new and emerging respiratory viruses with pandemic potential.
Virological data are collected through continuous global surveillance to monitor the characteristics of circulating influenza viruses, for example, in virus antigenicity, genetics and antiviral susceptibility to guide appropriate response and intervention activities. Data are used to inform the selection of the best candidate vaccine strains for the annual influenza vaccine. The surveillance activities inform a proportionate response that can be escalated in a rapid and coordinated manner as required.

The influenza laboratory network in Europe is well established and has performed virological surveillance since 1952 through World Health Organization (WHO)recognised national influenza centres (NICs) [7]. The European Influenza laboratory network was further strengthened in 1995 by the formation of the European Influenza Surveillance System (EISS) [8], which in 2003 became the Community Network of Reference Laboratories for Human Influenza in Europe (CNRL) [9]. Since then, the European Influenza laboratory network has been coordinated by the European Centre for Disease Prevention and Control (ECDC) and the WHO Regional Office for Europe (WHO/Europe) with the assistance of a group of experts from within the network, and is now termed the European Reference Laboratory Network for Human Influenza (ERLI-Net). The key capabilities of this network within Europe currently include:

- ability to respond to new and emerging influenza viruses through the development of laboratory diagnostic capabilities and data capture systems that link to clinical surveillance;

- provision of an appropriately trained cohort of virologists/microbiologists with the necessary laboratory surveillance techniques, in particular skills in virus detection and virus isolation; 
Key laboratory activities and outputs and the resulting public health outcomes for each strategic objective

Strategic objectives
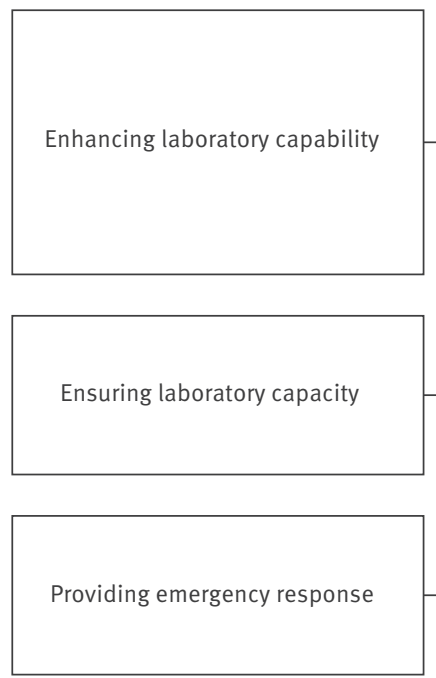

Translating laboratory data into information for public health action
Key laboratory activities and outputs

- Maintain and improve technical competence

- Implement new technologies

- Improve the data available to link severity and virus evolution

- Improve harmonisation and interpretation of antiviral susceptibility data

- Undertake a programme of training and quality assessment

- Improve the ability to produce or access reference/control material and reagents
- Optimise the representativeness of the surveillance system by using statistical sample size approach

- Improve the range of virus diagnosis (for other respiratory viruses)

- Incorporate flexibility and resilience for surge response
Public health outcomes

- Strain selection data for vaccine
production
- Recommendations on antiviral
susceptibility in different patient
subgroups; guidance on prophylaxis,
treatment and outbreak management in
complex clinical situations
- Improved knowledge about risk factors
for severe outcome of infection
- Improved estimation of disease burden
through virus detection in subgroups of
the population
- Increased flexibility of surveillance
systems to respond to evolving
situations
- Improved ability to detect, assess and
respond to respiratory virus threats in
near real-time
- Provision of timely scientific data to
inform disease prevention and control
policies and public health decision-making

- Inform disease control measures

- Improve communication and coordination of response for seasonal influenza and emergency incidents/situations

- Improve stakeholder liaison and communication
- Improve risk assessment and early warning response

- Improve ability to respond to emergent infections, including

provision of reagents

Increase collaborative links with other sectors
- generation of genetic and antigenic data on circulating influenza viruses;

- generation of phenotypic and genotypic data on influenza antiviral susceptibility;

- capability and capacity to respond to a rapid upsurge in laboratory activity as a result of an epidemic or pandemic of influenza viruses, with resilience to sustain a long-term response to an emerging threat;

- participation in regular external quality assurance (EQA) activities, underpinned by targeted training where necessary, to ensure reliability of results generated for diagnostic and surveillance purposes.

Changing health service priorities and political structures within the WHO European Region mean it is timely to consider the alignment of key laboratory networks and their future roles, to ensure maximum benefit from specialist activities.

\section{Objectives of this analysis}

To help align the diverse priorities of ECDC and WHO/ Europe, the development of common strategic objectives was undertaken to provide a framework for the maintenance and further development of a high-quality, cohesive laboratory network for the virological surveillance of influenza within the European Region. Strategic priorities were developed through consultation and a Delphi structured communication process over a period of six months in 2013 involving ERLI-Net members. This was used to further refine the strategic priorities before submission to ECDC and WHO/Europe.
Strategic priorities for the European influenza laboratory network

While national influenza laboratory surveillance systems across Europe differ in their foundation, organisation, funding basis and regional interaction with national authorities, the influenza virological surveillance activities undertaken by NICS are largely consistent.

Laboratory activities were categorised into four key strategic objectives in order to provide a framework to guide laboratories in prioritising their influenza surveillance activities, and to aid the identification of operational issues and challenges for the maintenance and development of the laboratory network in order to meet and adapt to new challenges, including the emergence of other respiratory virus threats, over the coming years: (i) enhancing laboratory capability; (ii) ensuring laboratory capacity; (iii) providing emergency response; and (iv) translating laboratory data into information for public health action.

The key laboratory activities and outputs, and the resulting public health outcomes in each of the strategic objectives are illustrated in Figure 1 . The priority areas for development in each strategic objective are shown in the Table.

\section{Enhancing laboratory capability}

The key priorities in ensuring that the laboratory network has the capability to characterise unusual influenza A viruses, send isolates for vaccine strain selection to the WHO Collaborating Centre for Reference and Research on Influenza, London, and operate under 


\begin{tabular}{|c|c|}
\hline Strategic objectives & Suggested actions in priority areas \\
\hline Enhancing laboratory capability & $\begin{array}{l}\text { - Undertake gap analysis to identify network requirements and develop workplan of remedial actions. } \\
\text { - Improve coordination of training activities between WHO/Europe and ECDC. } \\
\text { - Ensure expertise and technical support for developments so that network is responding to the latest } \\
\text { technological advances (e.g. generic PCRs, multiplex-PCRs, next generation sequencing, point-of-care } \\
\text { testing). } \\
\text { - Develop structured training activities to (i) address performance issues identified through EQA and (ii) } \\
\text { provide refresher/advanced training to laboratories with more established capabilities. } \\
\text { - Align laboratory core competences based on expert consultation of technology feasibility and network } \\
\text { - priorities for public health benefit. } \\
\text { - Improve the completeness and quality of antiviral susceptibility data reporting to inform use of } \\
\text { - Deviviral therapies and clinical guidance. } \\
\text { within the network in the event of an emerging virus. }\end{array}$ \\
\hline Ensuring laboratory capacity & $\begin{array}{l}\text { - Optimise the representativeness of country-level surveillance systems, e.g. using statistical sample } \\
\text { size. } \\
\text { - Ensure robust virological data from a range of healthcare settings e.g. primary care, secondary care } \\
\text { (both routine hospital admissions and critical care), and from a spectrum of clinical illness from } \\
\text { subclinical (through population-based surveillance studies and serological assessment) to severe } \\
\text { illness (SARI) and death. } \\
\text { - Virologists from network laboratories should be encouraged to liaise with their epidemiology } \\
\text { colleagues to develop plans for statistical and systematic approaches to support evidence-based } \\
\text { decisions. }\end{array}$ \\
\hline Providing emergency response & $\begin{array}{l}\text { - Improve risk assessment and early warning response, e.g. in the event of the emergence of a new virus } \\
\text { subtype, it may be necessary to quickly modify the sampling strategy to target population subgroups } \\
\text { preferentially affected (e.g. young children or pregnant women) to obtain data to inform the response. } \\
\text { - Effective use of virological data to enable early recognition of infection threats or prediction of } \\
\text { epidemiological trends, and facilitate early public health intervention. } \\
\text { - Incorporate flexibility and resilience for surge response with respect to diagnostics and workforce. } \\
\text { - Assess current status of BSL-3 facilities within the laboratory network, and develop plan to ensure all } \\
\text { countries have access to BSL-3 facilities to respond to highly pathogenic influenza virus strains. } \\
\text { - Develop a European laboratory network outbreak/emergency response plan so that network members } \\
\text { are aware of coordination arrangements and what outputs can be expected. } \\
\text { - Assessment and further development of laboratory testing algorithms. }\end{array}$ \\
\hline
\end{tabular}

BSL-3: biosafety level 3; ECDC: European Centre for Disease Prevention and Control; EQA: external quality assessment; PCR: polymerase chain reaction; SARI: severe acute respiratory illness; WHO/Europe: World Health Organization Regional Office for Europe.

biosafety level 3 (BSL-3) conditions to detect highly pathogenic influenza viruses, as well as other emerging respiratory virus threats such as MERS-CoV, are as follows. First, maintain technical skills such as virus isolation in embryonated chicken eggs and antigenic characterisation of virus isolates that are beginning to decline due to a shift to molecular technologies. Second, increase laboratory preparedness and resilience through the introduction of new capabilities such as molecular detection, sequencing and next generation sequencing.

To meet the diverse requirements of the European Region, where laboratories are geographically separated and developments are often undertaken at different speeds, a coordinated programme of training is required including the following: 'wet laboratory' practical training; a shift towards e-learning and distancelearning technologies; annual meetings; twinning and use of EQA to identify gaps and weaknesses in capability.

The rate of introduction of new skills into the laboratory network is dependent on underlying funding mechanisms and decisions about which capabilities to introduce versus which to omit must be undertaken at a strategic level.

Initiatives have been under way within ERLI-Net over recent years to offer network members a comprehensive programme of laboratory training, including practical-based training underpinned by theoretical instruction (for example, influenza virus isolation and characterisation and antiviral susceptibility testing), lecture-based theoretical instruction (for example, influenza sequencing) and more recently, e-learning webcast (for training sessions on primer design). A twinning initiative has been established to foster interaction and support between laboratory network members, supported by a small financial grant to cover 
Improving the capability for virus diagnosis within the European influenza laboratory network

$$
\begin{aligned}
& \text { Emerging new virus } \\
& \text { Viral sequences available } \\
& \text { Sharing of protocols }
\end{aligned}
$$

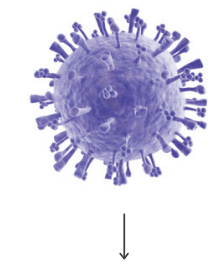

Laboratory network in silico assessment of typing and subtyping capability

Prediction of individual laboratory detection capability

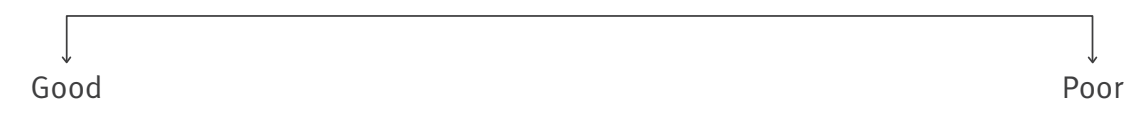

No further action

Adjust reagents

Wait for practical testing via distribution of viral RNA control material

Evaluation of testing results

\begin{tabular}{ccc}
\hline & $\downarrow$ & \\
$\begin{array}{l}\text { Recommendations } \\
\text { for improvements }\end{array}$ & Assessment of laboratory network preparedness & Distribution \\
of guidance
\end{tabular}

Source of image: (Olstockphoto

travel and subsistence costs to visit a partner laboratory. It is hoped that long-standing collaborations will be established between network laboratories to help improve resilience and communication.

Both WHO/Europe and ERLI-Net coordinate regular EQA programmes to evaluate the technical capability of the influenza network of laboratories. ERLI-Net undertakes biennial EQA of laboratory capability in European Union (EU)/European Economic Area (EEA) countries, results of which inform a programme of training activities. Through the regular collection of EQA data for the ERLINet, it is possible to undertake comparative analyses and identify changes in performance. The most recent EQA in 2013 included virus isolation and antigenic and genetic characterisation, and phenotypic and genetic characterisation of antiviral susceptibility techniques, which are not currently covered by the WHO EQA Project (EQAP) [10]. The 2011 antiviral EQA assessment by ERLINet was the first of its kind globally [11].

Laboratory preparedness and technical performance has also been assessed, through an 'in silico' exercise in 2011 to detect novel reassortant and circulating triple reassortant (TRA) influenza $A\left(\mathrm{H}_{3} \mathrm{~N}_{2}\right)$ swine viruses in humans [12], and more recently, in 2013, in collaboration with ECDC and WHO/Europe, for the rapid assessment of laboratory preparedness for detection of the novel avian influenza $A\left(\mathrm{H}_{7} \mathrm{~N} 9\right)$ virus in $\mathrm{EU} / \mathrm{EEA}$ countries [13].

In the future, rapid assessment activities, as illustrated in Figure 2, should be extended to all laboratories in the European Region to provide assurance on the range of virus diagnosis.

\section{Ensuring laboratory capacity}

The key priorities in ensuring capacity of laboratories in the network include ensuring robust staffing mechanisms and succession planning are in place, and ensuring the capacity to scale up the surveillance response and link to clinical surveillance programmes.

Network activities need to focus on staff succession planning to ensure that the European influenza laboratory network has the capacity to meet the shifting technological priorities. Surveillance development initiatives need to link virological data to clinical surveillance programmes, and be underpinned by robust statistical data to ensure the network will target its 
resources in the most efficient way, help inform effective intervention strategies, and provide a scaleable response in the event of a future epidemic or pandemic.

\section{Providing emergency response}

To provide timely and effective emergency response, the European influenza laboratory network needs to have coordinated arrangements for risk assessment and early warning.

Following the emergence of the influenza $A\left(\mathrm{H}_{7} \mathrm{~N} 9\right)$ virus in early 2013, ECDC, WHO/Europe and the ERLINet Coordination Team convened regularly by teleconference to plan activities to assess network capability, distribute positive-control viruses and virus RNA, provide relevant information on primers/probes for diagnosing influenza A viruses with pandemic potential, and to draft a technical briefing note on diagnostic preparedness [14]. Since then, regular communication has taken place between all partner organisations as part of early warning and response activities.

\section{Translating laboratory data into improved public health outputs}

The influenza laboratory network needs to maximise the public health outputs from routinely generated virological surveillance data. The priorities include: (i) targeted surveillance of deaths and serious illness due to influenza; (ii) estimating influenza vaccine effectiveness and identifying influenza vaccine failures; (iii) timely analysis and interpretation of data to inform public health action; and (iv) improved technical data provision through improved visual display and dissemination.

A number of initiatives are already under way to use the wealth of virological data generated by the influenza laboratory network to strengthen evidence-based decision-making: for example, some laboratories currently undertake surveillance for severe disease and deaths due to influenza (SARI surveillance). These data are incorporated into the routine weekly influenza surveillance overview (WISO) produced by ECDC [15]. Further work is underway to improve the quality and comparability of SARI surveillance data across the European region [16], and increase the number of countries who currently participate.

ERLI-Net produces regular influenza virus characterisation surveillance reports for ECDC, which present the results of the antigenic and genetic analysis of influenza viruses isolated in Europe undertaken by WHO Collaborating Centre for Reference and Research on Influenza in London [17]. The reports provide network virologists with detailed technical data that allow them to compare viruses they have isolated in a wider background context. This may help assist evaluation of circulating strains within different countries.

A single software platform was implemented in the 2014/15 influenza season for data collection across the whole European region, rather than the previous separate ECDC- and WHO/Europe-run systems. Data collected via this platform have been used to produce a single joint surveillance bulletin [18], thereby improving consistency in communication across the region.

\section{Conclusions}

Successful implementation of these strategic objectives will produce a European laboratory network that can deliver the following:

- the capability and capacity to monitor seasonal influenza and to respond to the emergence of novel influenza virus subtypes and reassortants, as well as other newly emerging respiratory virus infections in a timely and coordinated manner;

- a scalable response in the event of a newly emerging influenza virus or other novel respiratory virus threat;

- expertise and technical developments to ensure that it is responding to the latest technological advances (e.g. generic PCRs, multiplex PCRs, next generation sequencing, point-of-care testing);

- outputs required by stakeholders to inform public health action;

- the provision of timely and accurate surveillance data to health professionals and commissioners of healthcare services to inform effective intervention (e.g. vaccination programmes or antiviral treatment) and improve patient outcomes;

- a programme of EQA to ensure the technical capability of laboratories within the network;

- a programme of training and development for virologists within the network to maintain technical competence and ensure continuing professional development;

- identification of areas for further research and development in diagnostics, surveillance and control of infection (through antiviral drugs and vaccination programmes).

Influenza laboratories across Europe operate in different contexts, for example, public health institutes, universities or research agencies, with little or no consistency in funding mechanisms. As a result, the laboratories use different technical approaches and diagnostic platforms according to local need, which is challenging for the standardisation of molecular activities in particular. As the financial pressures of the current economic climate are expected to continue over the coming years, a more systematic and strategic approach to virological surveillance is needed to respond to shifting priorities. 
Optimisation of surveillance systems using, for example, statistical sample size calculations, will provide a desired level of confidence in the data to inform situational assessments, improve system approaches, focus resources and justify funding needs. By enhancing the public health benefit of the data through timely reporting and analysis, it will be possible to strengthen the evidence-based decision-making to inform policymakers and disease prevention and control strategies within a country and across the wider European Region.

Strategic planning for the European influenza laboratory network coincides with ongoing surveillance development activities under way within Europe [19] and in the US [20]. A number of the activities outlined in the Table are already under way within ERLI-Net. In order to achieve efficient and effective implementation of the recommendations across the European Region, ECDC and WHO/Europe will need to develop a systematic approach to assessing current laboratory capability and capacity, and provide the tools necessary to aid planning and resource allocation within defined timescales. This will ensure that the network is able to respond in a strategic and efficient manner to new challenges over the next 3-5 years and develop a longer-term strategy over the next 5-10 years, thereby contributing effectively to the global surveillance of influenza and other emerging respiratory virus infections.

\section{Acknowledgements}

Discussions around the strategic development of the European influenza laboratory network have taken place during a number of workshops and meetings convened by ECDC and WHO/Europe. We are grateful to members of the European influenza laboratory network for contributing to the discussions, and in particular to Eeva Broberg, Cornelia Adlhoch and Pasi Penttinen at ECDC, and Caroline Brown and Dmitriy Pereyaslov at WHO/Europe for commenting on drafts of the paper.

Work at the MRC National Institute for Medical Research was supported through MRC programme number U117512723.

\section{Conflict of interest}

None declared.

\section{Authors' contributions}

MZ, NG and HRA initiated the discussions on strategic development; all co-authors were involved in subsequent discussion and development of key strategic development areas. NG and HRA presented the strategy at European influenza network workshops and meetings and facilitated discussion with network members. NG drafted the initial manuscript with HRA; all co-authors reviewed and commented including approval of the final version.

\section{References}

1. Novel Swine-Origin Influenza $A\left(\mathrm{H}_{1} \mathrm{~N}_{1}\right)$ Virus Investigation Team, Dawood FS, Jain S, Finelli L, Shaw MW, Lindstrom S, et al. Emergence of a novel swine-origin influenza $A\left(\mathrm{H}_{1} \mathrm{~N}_{1}\right)$ virus in humans. N Engl J Med. 2009;36o(25):2605-15.http://dx.doi. org/10.1056/NEJMoa0903810 PMID:19423869

2. Jhung MA, Epperson S, Biggerstaff M, Allen D, Balish A, Barnes $\mathrm{N}$, et al. Outbreak of variant influenza $A\left(\mathrm{H}_{3} \mathrm{~N}_{2}\right)$ virus in the United States. Clin Infect Dis. 2013;57(12):1703-12.http:// dx.doi.org/10.1093/cid/cit649 PMID:24065322

3. Meng Z, Han R, Hu Y, Yuan Z, Jiang S, Zhang X, et al. Possible pandemic threat from new reassortment of influenza $A\left(\mathrm{H}_{7} \mathrm{Ng}\right)$ virus in China. Euro Surveill. 2014;19(6). PMID:24556346

4. Ren L, Yu X, Zhao B, Wu F, Jin Q, Zhang X, et al. Infection with possible precursor of avian influenza $\mathrm{A}\left(\mathrm{H}_{7} \mathrm{~N} 9\right)$ virus in a child, China, 2013. Emerg Infect Dis. 2014;20(8):1362-5.http://dx.doi. org/10.3201/eid2008.140325 PMID:25061975

5. Chen H, Yuan H, Gao R, Zhang J, Wang D, Xiong Y, et al. Clinical and epidemiological characteristics of a fatal case of avian influenza A H10N8 virus infection: a descriptive study. Lancet. 2014;383(9918):714-21.http://dx.doi.org/10.1016/S01406736(14)60111-2 PMID:24507376

6. Bermingham A, Chand MA, Brown CS, Aarons E, Tong C, Langrish C, et al. Severe respiratory illness caused by a novel coronavirus, in a patient transferred to the United Kingdom from the Middle East, September 2012. Euro Surveill. 2012;17(40):20290. PMID:23078800

7. Kitler ME, Gavinio P, Lavanchy D. Influenza and the work of the World Health Organization. Vaccine. 2002;20(Suppl 2):S5-14.http://dx.doi.org/10.1016/So264-410X(02)00121-4 PMID:12110248

8. Fleming DM, Cohen JM. Experience of European collaboration in influenza surveillance in the winter of 1993-1994. I Public Health Med. 1996;18(2):133-42.http://dx.doi.org/10.1093/ oxfordjournals.pubmed.a024472 PMID:8816310

9. Meijer A, Valette M, Manuguerra JC, Pérez-Breña P, Paget J, Brown C, et al.; Virology Working Group of the European Influenza Surveillance Scheme. Implementation of the community network of reference laboratories for human influenza in Europe. J Clin Virol. 2005;34(2):87-96.http:// dx.doi.org/10.1016/j.jcv.2005.02.005 PMID:16157259

10. World Health Organization (WHO). WHO External Quality Assessment Project for the detection of influenza virus type A by PCR. Geneva: WHO. [Accessed 17 Dec 2014]. Available from http://www.who.int/influenza/gisrs_laboratory/ external_quality_assessment_project

11. Thompson CI, Lackenby A, Daniels RS, McCauley JW, Pereyaslov D, Broberg EK, et al. Evaluation of influenza virus antiviral susceptibility testing in Europe: results from the first external quality assessment exercise. J Clin Virol. 2013;56(3):212-8.http://dx.doi.org/10.1016/j.jcv.2012.11.005 PMID:23201459

12. European Centre for Disease Prevention and Control (ECDC). CNRL in silico exercise to determine the capabilities of network laboratories to detect triple reassortant swine origin influenza $\mathrm{A}\left(\mathrm{H}_{3} \mathrm{~N}_{2}\right)$ viruses. Stockholm: ECDC; 2012 Available from: http:// ecdc.europa.eu/en/publications/Publications/1204-TER-CNRLCapability-Exercise.pdf

13. Broberg E, Pereyaslov D, Struelens M, Palm D, Meijer A, Ellis $J$, et al. Laboratory preparedness in EU/EEA countries for detection of novel avian influenza $A\left(\mathrm{H}_{7} \mathrm{Ng}\right)$ virus, May 2013. Euro Surveill. 2014;19(4):20682. PMID:24507469

14. European Centre for Disease Prevention and Control (ECDC). Diagnostic preparedness in Europe for detection of avian influenza $\mathrm{A}\left(\mathrm{H}_{7} \mathrm{~N} 9\right)$ viruses. Technical briefing note, 23 April 2013. Stockholm: ECDC;2013. Available from: http://www.ecdc.europa.eu/en/ publications/layouts/forms/Publication DispForm. aspx? List=4f55ad51-4aed-4d32-b960-af70113dbb9o\&ID=789

15. European Centre for Disease Prevention and Control (ECDC). Weekly influenza surveillance overview. Stockholm: ECDC. [Accessed 17 Dec 2014]. Available from: http://www.ecdc. europa.eu/en/publications/surveillance_reports/influenza/ Pages/weekly_influenza_surveillance_overview.aspx

16. Beaute J. Evaluation of severe influenza surveillance in Europe. 4th Joint WHO/Europe-ECDC Annual Influenza Surveillance Meeting, 11-13 June 2014, Vienna, Austria. Slides available from: http://pt.slideshare.net/ECDC_EU/ evaluation-of-severe-influenza-surveillance-in-europe

17. European Centre for Disease Prevention and Control (ECDC). Influenza virus characterisation. Stockholm: ECDC. [Accessed 17 Dec 2014]. Available from: http://www.ecdc.europa.eu/en/ publications/surveillance_reports/influenza/Pages/influenza virus_characterisation.aspx

18. European Centre for Disease Prevention and Control (ECDC)/ WHO Regional Office for Europe (WHO Europe). Flu News Europe. Stockholm/Paris: ECDC/WHO Europe. [Accessed 17 Dec 2014]. Available from: http://flunewseurope.org

19. European Centre for Disease Prevention and Control (ECDC). Core functions of microbiology reference laboratories 
for communicable diseases. Stockholm: ECDC; 2010.

Available from: http://ecdc.europa.eu/en/publications/

Publications/1006_TER_Core_functions_of_reference_labs.pdf

20. Association of Public Health Laboratories (APHL). Influenza

virologic surveillance right size road map. Silver Spring,

MD: APHL; 2013. Available from: http://www.aphl.org/

aphlprograms/infectious/influenza/Pages/Influenza-Virologic-

Surveillance-Right-Size-Roadmap.aspx 